\title{
Mein Buch
}

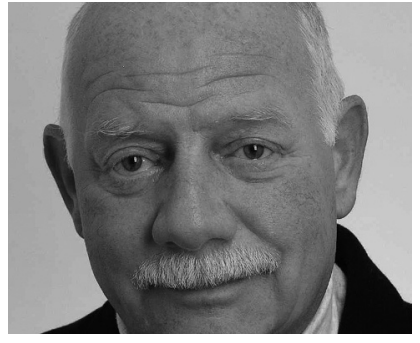

\section{VON RAINER KNAPP}

Rainer Knapp ist Techniker und Betriebswirt. Nach verschiedenen Tätigkeiten in Technik, Vertrieb und Management war er von 1975 bis Mitte 2010 Geschäftsführer der GWW Gemeinnützige Werkstätten und Wohnstätten $\mathrm{GmbH}$ in Gärtringen (BadenWürttemberg). Ehrenamtlich war er bis zu seiner Pensionierung in verschiedenen Verbandsgremien der Lebenshilfe und der Bundesarbeitsgemeinschaft Werkstätten für behinderte Menschen tätig. Seit seinem Ruhestand Jahr betätigt er sich als selbständiger Berater für Organisations- und Managementfragen und ist im Krisenmanagement und in der Sanierung von Unternehmen tätig.

E-Mail

Rainer.Knapp@femos-zenit.de
Klaus Dörner stellt einst die Frage: »Was machen wir mit denen, die nicht so sind wie wir, deren Leistungswert sie (industriell) unbrauchbar macht; wofür sind sie da und wie gehen wir mit ihnen um? «(Dörner 1988)

Als ich 1975, mit 30 Jahren gerade langsam erwachsen werdend und durch Ausbildung und bisherige berufliche Tätigkeit auf Rationalität und Effizienz gepolt, die Geschäftsführung einer neugegründeten Behinderteneinrichtung übernahm, war ich zunächst heftig irritiert.

In der »Szene « wahrgenommene unklare Interessenlagen, wohlwollende Fremdbestimmtheit, nicht überprüfbare soziale Absichtserklärungen und der Widerspruch zwischen sozialen Postulaten und realem Handeln machten mir bewusst, dass ich, wenn ich meiner Aufgabe verantwortlich und verantwortungsvoll gerecht werden wollte, zunächst für mich diese Frage von Klaus Dörner beantworten und mir über meine eigenen Wertvorstellungen im Klaren sein muss.

Von vielen guten und hilfreichen Büchern war und ist für mich das Buch von Jürgen Reyer »Alte Eugenik und Wohlfahrtspflege - Entwertung und Funktionalisierung der Fürsorge vom Ende des 19. Jahrhunderts bis zur Gegenwart « eine unentbehrliche Grundlage einer unverzichtbaren und dauerhaft notwendigen Wertediskussion in Bezug auf den Umgang unserer Gesellschaft mit dem »Anderssein ", mit ungeprüften Normsetzungen und ökonomischen Nützlichkeitspostulaten.

Erste noch gutgemeinte eugenische Ansätze entwickeln sich, einer nicht wertegebundenen und scheinrationalen Logik fol- gend, zu Akzeptanz und Befürwortung rassehygienischer Konzepte bis hin zur Euthanasie auch durch diejenigen, denen Schutz und Fürsorge die betroffenen behinderten Menschen anvertraut waren.

Diese Entwicklung erschreckt und bedrückt auch, scheint sie doch, wie im abschließenden Kapitel »Neue Eugenik und Soziale Arbeit « fundiert dargestellt, wenn auch mit anderen Vorzeichen wiederholbar zu sein.

Das Buch von Reyer hilft, die Dynamik von Entwicklungen zu verstehen, zwingt zu der Überprüfung der eigenen Positionen und macht deutlich, dass ohne eine verbindliche ethische Grundlage der Gleichwertigkeit aller behinderten Menschen (und nicht nur diese) Gefahr laufen, ökonomischen Nützlichkeitsbewertungen und eugenischen Idealphantasien (mit allen Folgen) ausgesetzt zu werden.

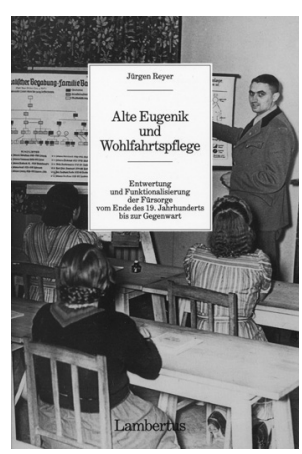

Jürgen Reyer: Alte Eugenik und Wohlfahrtspflege. Entwertung und Funktionalisierung der Fürsorge vom Ende des 19. Jahrhunderts bis zur Gegenwart. Lambertus-Verlag, Freiburg im Breisgau. 264 Seiten. 14,40 Euro. ISBN 978-3-7841-0506-2. 